\title{
Thermomechanical cyclic behavior modeling of SMA materials and structures
}

\author{
Shabnam Arbab Chirani ${ }^{1,2, a}$, Luc Saint-Sulpice ${ }^{1,2}$, and Sylvain Calloch ${ }^{2}$ \\ 1 Université Européenne de Bretagne \\ Ecole Nationale d'Ingénieurs de Brest \\ Laboratoire Brestois de Mécanique et des Systèmes (EA 4325) \\ Technopôle Brest-Iroise, CS 73862, 29238 Brest Cedex 3, France \\ 2 Université Européenne de Bretagne \\ Ecole Nationale Supérieure d'Ingénieurs \\ Laboratoire Brestois de Mécanique et des Systèmes (EA 4325) \\ 2 rue François Verny, F-29806 Brest Cedex 9, France
}

\begin{abstract}
A 3D thermomechanical macroscopic model describing the cyclic effects in SMA has been developed by Sant-Sulpice et al.. The essential of this model is based on the introduction of a variable describing the development of jammed martensite. As it is known, a residual strain under cyclic thermomechanical loadings is developed in SMA like the ratcheting effect in plasticity. The origin of the evolution of the residual strain in SMA can be the plasticity or the development of jammed martensite. In some SMAs, its origin has been verified and it has been concluded that it is mainly due to the jammed martensite. This permits to justify the models constitutive equations. The developed model has been validated by simple thermomechanical cyclic tests. It has been integrated numerically in Cast3M finite elements code. This study is organised in two parts. The first one concerns the numerical scheme and the validation tests on a volumic element under simple cyclic loadings, cyclic proportional and non proportional loadings. The second part concerns the numerical study of SMA structures. Two coil sprins actuators under cyclic assisted shape memory effect are also studied.
\end{abstract}

\section{Introduction}

More and more in applications SMA structures undergo the cyclic loadings. They present the fatigue effect because of the cyclic nature of the loading. This can create a cumulative residual strain leading to fatigue phenomenon $[1,2]$. This effect has been particularly studied in metallic alloys during the last twenty years [3,4]. But few studies concern the SMA [5]. It is so necessary to develop an adapted model for these alloys taking into account the cumulated residual strain, to ensure the reliability of the global structure. The first purpose of the present work is to propose a macroscopic model taking into account the particularities of SMA behavior under pseudoelasticity and thermomechanical loadings. It includes the return point memory effect, the tension-compression asymmetry and the residual deformation. For this, the model of Bouvet et al. [6] has been initially used and then modified. The important point in this model is the introduction of a particular kinetic of transformation strain which takes into account the evolution and the accumulation of residual strain during cyclic loadings based on the definition of jammed martensite. The realized experiments show that the jammed martensite seemed to be the origin of the residual strain in the studied alloy. The second purpose of the study is to

\footnotetext{
a e-mail: arbab@enib.fr
} 
show the numerical integration method and the results of finite elements simulations on SMA structures under cyclic loadings.

This paper is organized in three parts. The first one describes the constitutive equations of the model. The second part present the numerical integration of the model in a finite elements code. The third part concerns the simulation of a cyclic thermomechanical loading on two antagonistic actuators.

\section{3D macroscopic model for cyclic thermomechanical behavior of SMA}

The proposed model is based on the model recently proposed by Bouvet et al. [6] which permits to obtain the superelasticity of SMA under general multiaxial nonproportional loading. It has been explained in more details in $[7,8]$. The main originality concerns the description of the elastic domain of the material by two different transformation surfaces for forward and reverse transformation noted $f_{1}$ and $f_{2}$, respectively (Figure 1 ). Over and above the other particularities of the behavior, the definition of these two transformation surfaces permits to describe particularly the reorientation of martensite observed under the nonproportional loadings.

In this work, modifications are proposed to take into account the evolution of the residual strain due to residual martensite under cyclic loading. Particularly, the concept of cumulated transformation strain, $t r$, leading the evolution of jammed martensite volume fraction has been introduced in the model (See Equ. 7).

\subsection{Strain decomposition}

Beside the classical observable variables, the total strain, $\varepsilon$, and the temperature, $T$, the transformation strain tensor, $\varepsilon^{t r}$, is used as internal variable. The transformation strain tensor is defined by the strain decomposition

$$
\underline{\varepsilon}^{t r}=\underline{\varepsilon}-\underline{\varepsilon}^{e}=\underline{\varepsilon}-\underline{\underline{E}}^{-1} \underline{\sigma}
$$

Where $\underline{\varepsilon}^{e}$ is the elastic strain and $\underline{E}^{-1}$ is the fourth ordre Hook's tensor. To simplify the problem, the same elastic behavior is assumed for austenitic and martensitic phases. The martensite volume fraction, $z$, is defined by $[9]$ :

$$
z=\frac{\varepsilon_{e q}^{t r}}{\gamma}
$$

Where $\gamma$ is the maximum transformation strain and is a material parameter; the equivalent transformation strain, $\varepsilon_{e q}^{t r}$, is defined by [6].

\subsection{Description of the two transformation surfaces}

Two transformation surfaces have been introduced in the model. The first one, $f_{1}$, drives the forward transformation, and the second one, $f_{2}$, drives the reverse transformation. The intersection of the two surfaces defines the elastic domain of the biphased material (Figure 1, grayed zone). The shape of the forward transformation surface is given by the following transformation function:

$$
f_{1}=\bar{\sigma}-\frac{R(z)+\sigma_{0}}{g\left(y_{\sigma}\right)} \leq 0
$$

Where $\bar{\sigma}=\sqrt{\frac{3}{2} \underline{s}: \underline{s}}$, with $\underline{s}$ the deviator stress tensor, $g\left(y_{\sigma}\right)=\cos \left(\frac{\arccos \left(1-a\left(1-y_{\sigma}\right)\right)}{3}\right)$ describe the tensile-compression asymetry, $y_{\sigma}=27 \frac{\operatorname{det}(\underline{s})}{2 \bar{\sigma}^{3}}$ is the third invariant, $R(z)$ is the size 
of the transformation surface and $\sigma_{0}=\mu\left(T-T_{0}\right)$ is the transformation stress under tensile loading which depends on the temperature. The shape of the reverse transformation surface is the same:

$$
f_{2}=\overline{\sigma-X}-\frac{R(z)+\sigma_{0}}{g\left(y_{\sigma-X}\right)} \leq 0
$$

Where $\underline{X}$ determines the center of $f_{2}$.
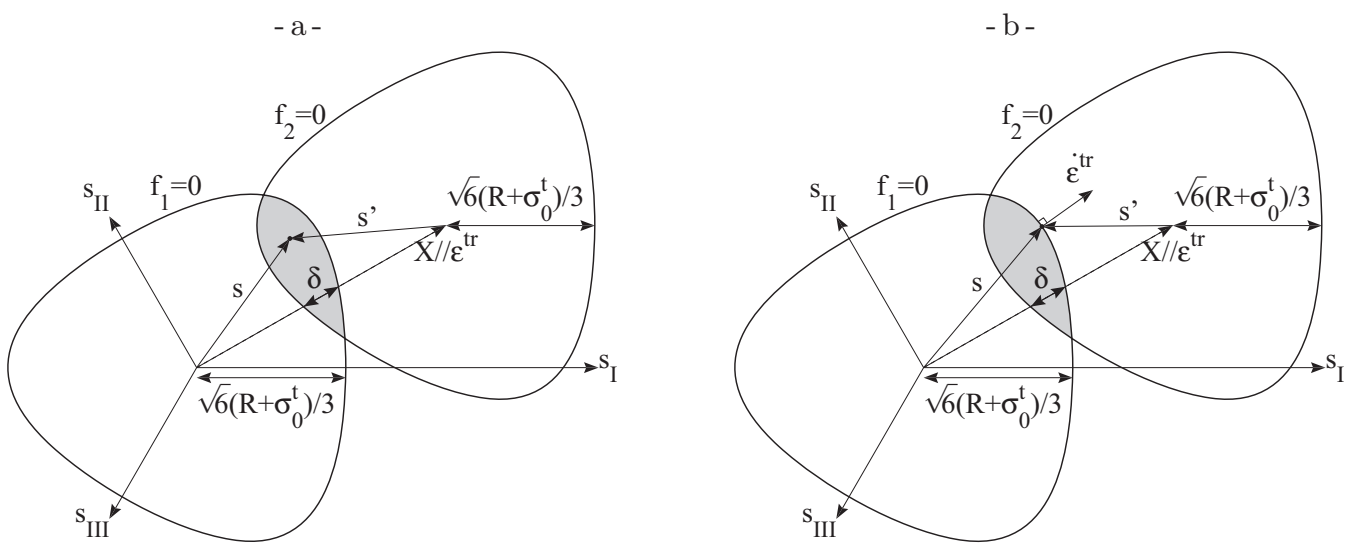

$c-$
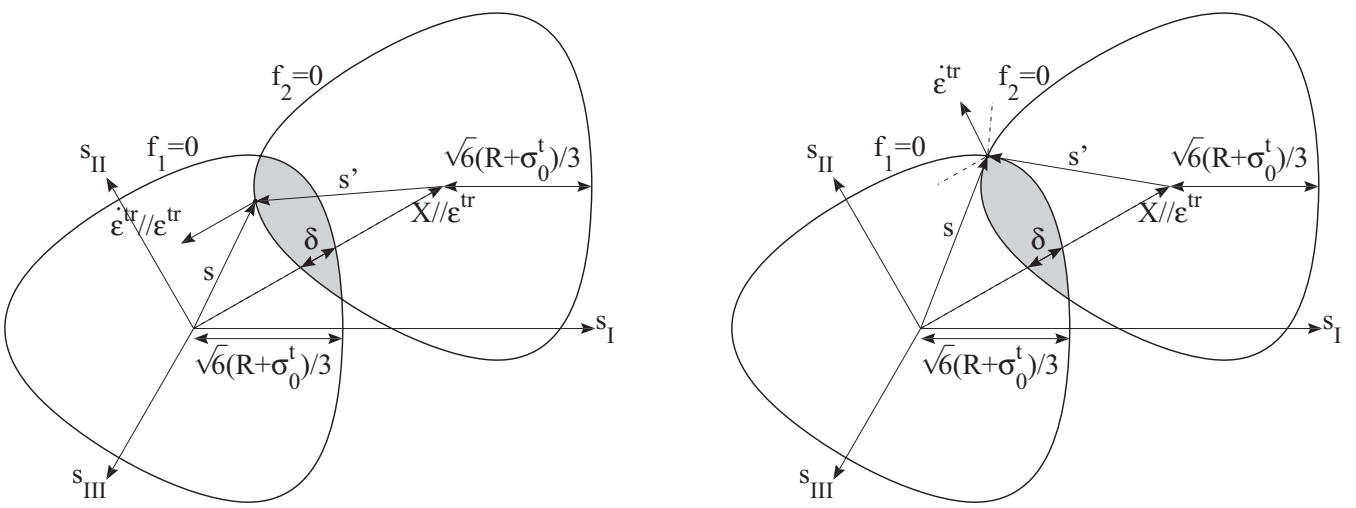

Fig. 1. Forward and reverse transformation surfaces during: a) elasticity of a biphased SMA, b) forward transformation, c) reverse transformation and d) reorientation of martensite.

\subsection{Transformation strain evolution}

The flow rules of the transformation strain, $\varepsilon^{t r}$, are given by equation (7). Concerning the forward transformation the normality law is respected, but during pure reverse transformation there is no any martensite reorientation. So the transformation strain direction remain the same. Consequently, a "non-associated" frame is defined. It means that the transformation direction is parallel to the transformation strain and not normal to the reverse transformation transformatin surface $\left(f_{2}\right)$. 


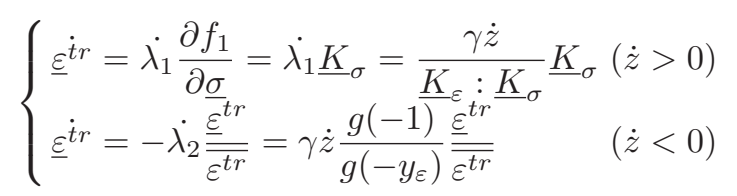

Where $\dot{\lambda_{1}}$ and $\dot{\lambda_{2}}$ are the transformation mutipliers given by the consistency conditions.

\subsection{Cyclic behavior}

The origin of the residual strain that appears during cyclic loadings as been determined as residual martensite [8]. So, in order to simulate the cyclic behavior we introduce a volume fraction of jammed marteniste, $z_{b}$, which leads to a residual strain:

$$
\underline{\varepsilon^{b}}=\frac{\gamma \dot{z_{b}}}{\underline{K}_{\varepsilon}: \underline{K}_{\sigma}} \frac{\partial f_{1}}{\partial \underline{\sigma}}=\frac{\gamma \dot{z_{b}}}{\underline{K}_{\varepsilon}: \underline{K}_{\sigma}} \underline{K}_{\sigma}
$$

The evolution of the volume fraction of jammed marteniste depends on the cumulated transformation, $t r$ :

$$
\begin{gathered}
t r=\int\left|\frac{d \varepsilon^{t r}}{\gamma}\right| \\
\dot{z}_{b}=\frac{\mathrm{e}^{B \Delta z} \mathrm{e}^{\frac{\mathrm{e}^{B \Delta z} \ln (1+t r)-z_{b}}{C}}}{1+t r}
\end{gathered}
$$

\section{Numerical integration}

The global numerical algorithm of the model is describe on the figure 2. It consist in :

1. an elastic prediction from the initial stress, $\sigma_{n}$ and the strain increment, $\underline{\Delta \varepsilon}$, imposed by the finite element code,

2. calculate the value of the forward and reverse transformation functions,

3. then four case may happen :

(a) if the induced stress leads to negative transformation functions, then the behavior is really the elasticity.

(b) if the forward transformation function is positive, then forward transformation happen.

(c) if the reverse transformation function is positive, then reverse transformation happen.

(d) if both transformation functions are positive, then the reorientation takes place.

As shown on the figure 3 , the reorientation is realised by alternating between forward and reverse transformation until forward and reverse transformation functions are both negative or zero.

The return-mapping algorithm is used for the numerical integration of the transformation process. It consist in realizing a first order approximation of the transformation function's evolution:

$$
f_{1}^{j+1}=f_{1}^{j}+\frac{\partial f_{1}^{j}}{\partial \underline{\sigma}}:\left(\underline{\sigma}^{j+1}-\underline{\sigma}^{j}\right)+\frac{\partial f_{1}^{j}}{\partial R}\left(R^{j+1}-R^{j}\right)
$$

This leads to the needed evolution of martensite volume fraction in order to reduce the transformation function's value to zero:

$$
\mathrm{d} z=\frac{-f_{1}^{j}}{\frac{\partial f_{1}^{j}}{\partial \underline{\sigma}}: \frac{\partial \underline{\sigma}}{\partial z}+\frac{\partial f_{1}^{j}}{\partial R} R^{\prime}(z)}
$$




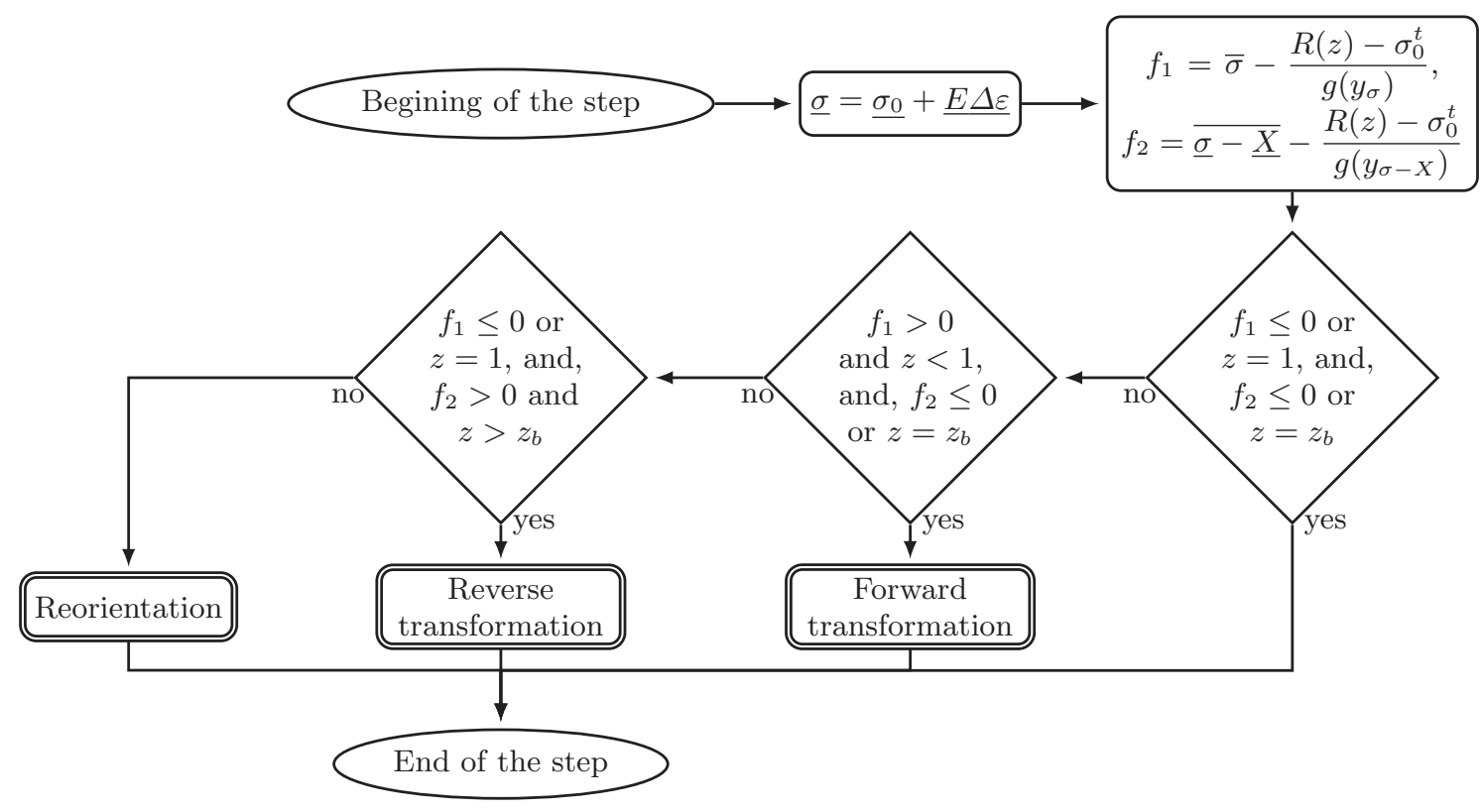

Fig. 2. Global flowchart of the numerical integration method used in the model.

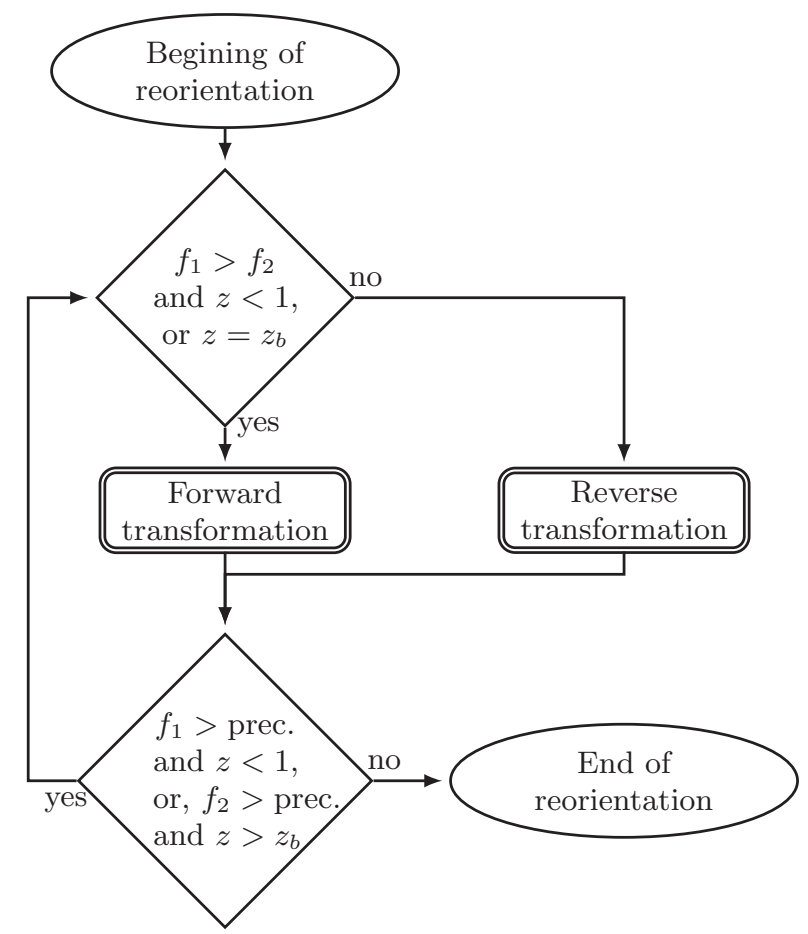

Fig. 3. Flowchart of the numerical integration of the reorientation.

As we used an approximation the transformation function's value will not necessarily be zero after this transformation correction. So this process must be repeated until the transformation function's value become smaller than a given precision (see Fig 4). 


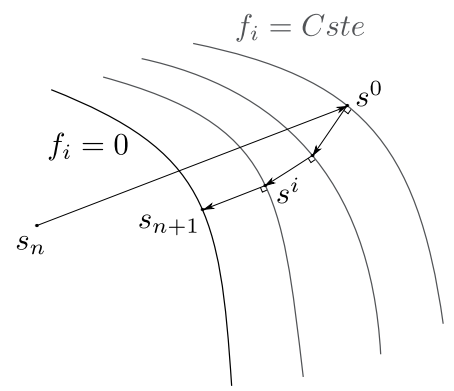

Fig. 4. Diagram of the deviatoric stress correction during a step of the numerical integration with the return-mapping method.

\section{Finite elements simulations}

First the behavior of the model has been validated by applying thermomechanical loadings to a elementary volume in a finite element code. The material parameters have been determined with tensile experiments on a Cu-Al-Be SMA (see Tab. 1).

Table 1. Material parameters used for the simulations.

\begin{tabular}{llllll}
\hline$\gamma$ & $a$ & $\mu$ & $T_{0}$ & $B$ & $C$ \\
\hline $5.85 \%$ & 0.7 & 0.3 & $-31^{\circ} \mathrm{C}$ & 5.02 & 0.0637 \\
\hline
\end{tabular}

The results of the two simulations are given on the figure 5. We observe the residual strain after unloading or heating due to the jammed martensite.
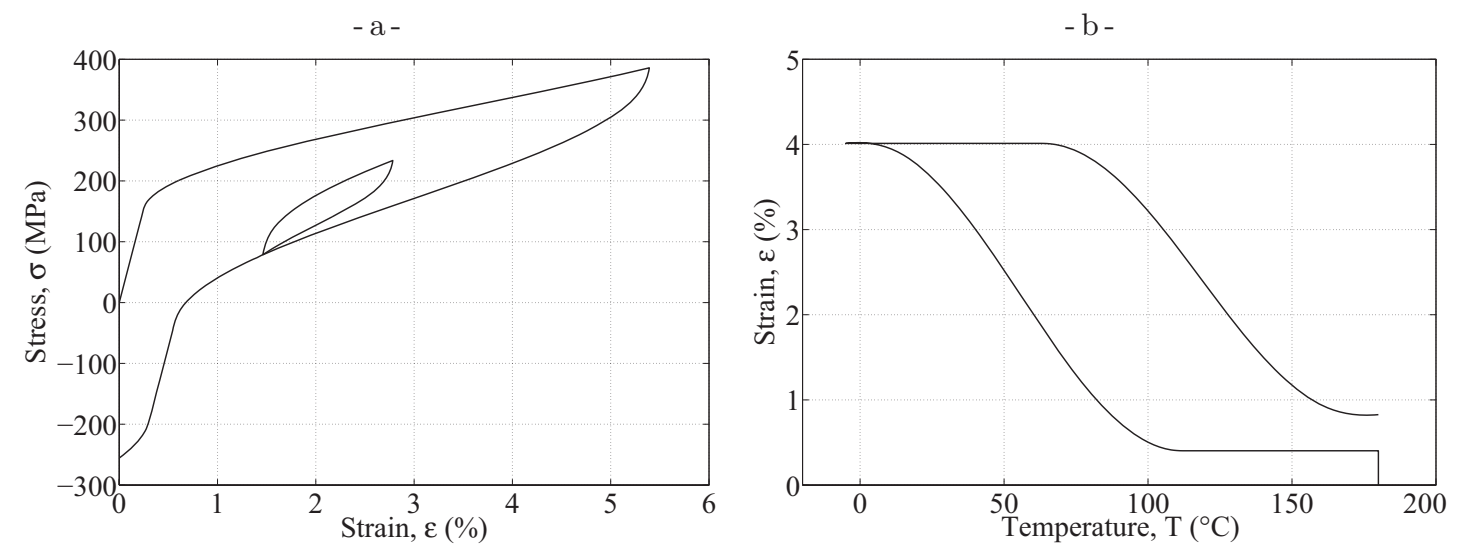

Fig. 5. Evolution of: a) the stress versus the strain and b) the strain versus the temperature, during two simulations of thermomechanical loadings.

Secondly, two actuators made up of two coil springs (see Fig. 6) have been studied. Each spring is composed of four turns with 30 sections of 48 elements. Then, one spring is made up of 5,760 elements. We used CUB20 elements (solid cubic elements with 20 nodes each) so it make a total of 26,321 nodes. The wire diameter is $3 \mathrm{~mm}$, the turns diameter is $20 \mathrm{~mm}$ and the pitch is $4 \mathrm{~mm}$. One end of the actuator is fixed, on the other end a fixed displacement is applied at 
high temperature in order to preload the actuator. The axial displacement of the central end of the springs is set to be the same on the two springs. We used two different actuators:

- The SMA/elastic actuator consist of one SMA spring on which we apply a cyclic thermal loading and an elastic spring which remains at room temperature.

- The SMA/SMA actuator consist of one SMA spring on which we apply a cyclic thermal loading and an similar SMA spring which remains at room temperature and works in pseudoelasticity. It explains the large deformation of this spring observed on figure 6 .
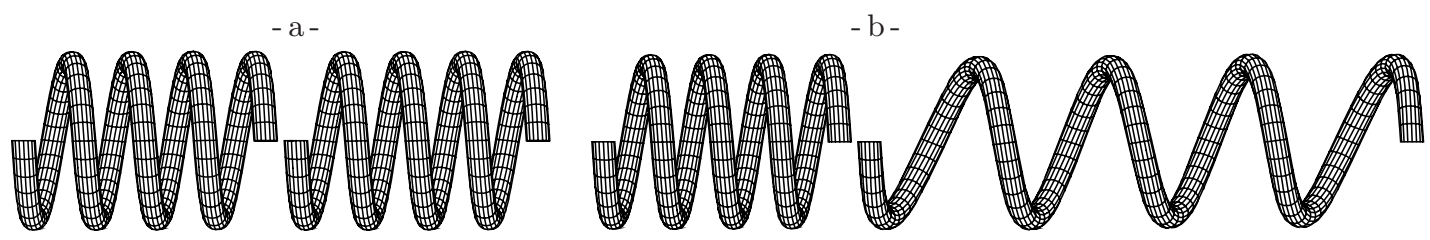

Fig. 6. Mesh of the coil springs from: a) the SMA/elastic actuator and b) the SMA/SMA actuator, after applying the preload.

The figure 7 shows the evolution of the actuator displacement during the cyclic thermal loading for the two actuators. We observe that the displacement for the SMA/SMA actuator is more than twice the one for the SMA/elastic actuator whereas the SMA/SMA actuator is only longer by an half. However there is more residual strain in the SMA/SMA actuator.
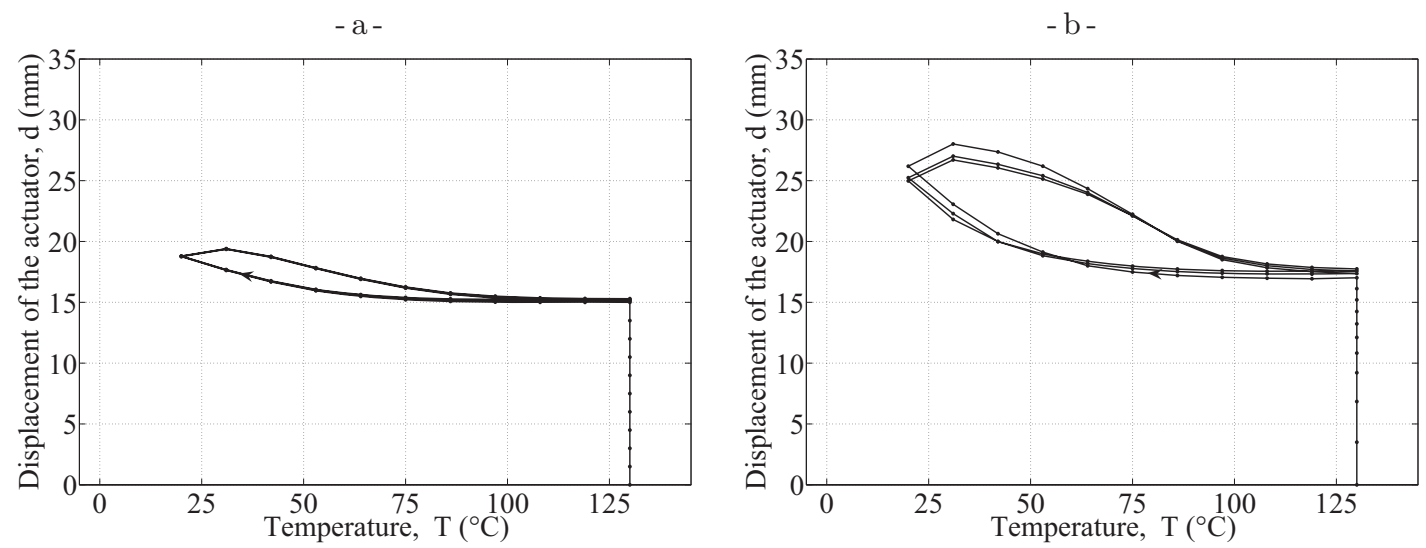

Fig. 7. Evolution of the actuator displacement versus of the temperature for: a) the SMA/elastic actuator and b) the SMA/SMA actuator.

The figure 8 shows the evolution of the force needed to maintain the fixed displacement during the cyclic thermal loading for the two actuators. We observe that the force decreases during the cooling due to the transformation strain.

The figure 9 presents the distribution of the martensite volume fraction in a section of the first spring of the actuators after the first cooling. We observe that the martensite volume fraction that appears is very similar for the two actuators.

The figure 10 shows the distribition of the equivalent stress in a section of the first spring of the SMA/SMA actuator before and after the first cooling. Due to the transformation a stress redistribution occurs: the stress at the outside of the section decreases while the stress near the center of the section increases. The distribition of the equivalent stress in a section of the first spring of the SMA/elastic actuator is almost identical. 

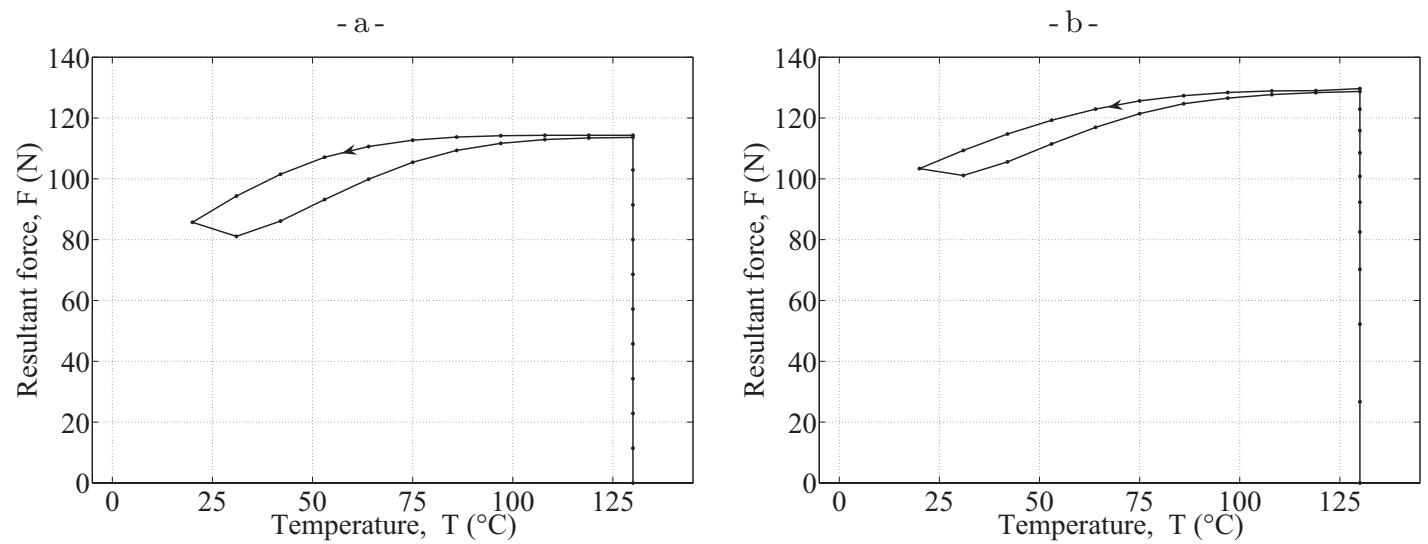

Fig. 8. Evolution of the force applied on: a) the SMA/elastic actuator and b) the SMA/SMA actuator, versus the temperature.
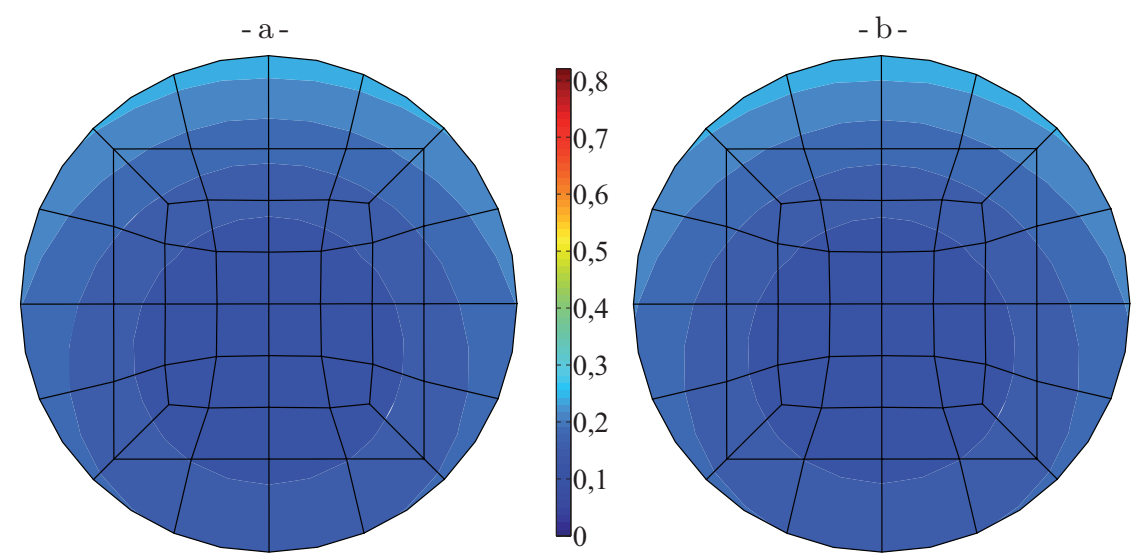

Fig. 9. Distribution of the martensite volume fraction after cooling in a section of the first spring of: a) the SMA/elastic actuator and b) the SMA/SMA actuator.
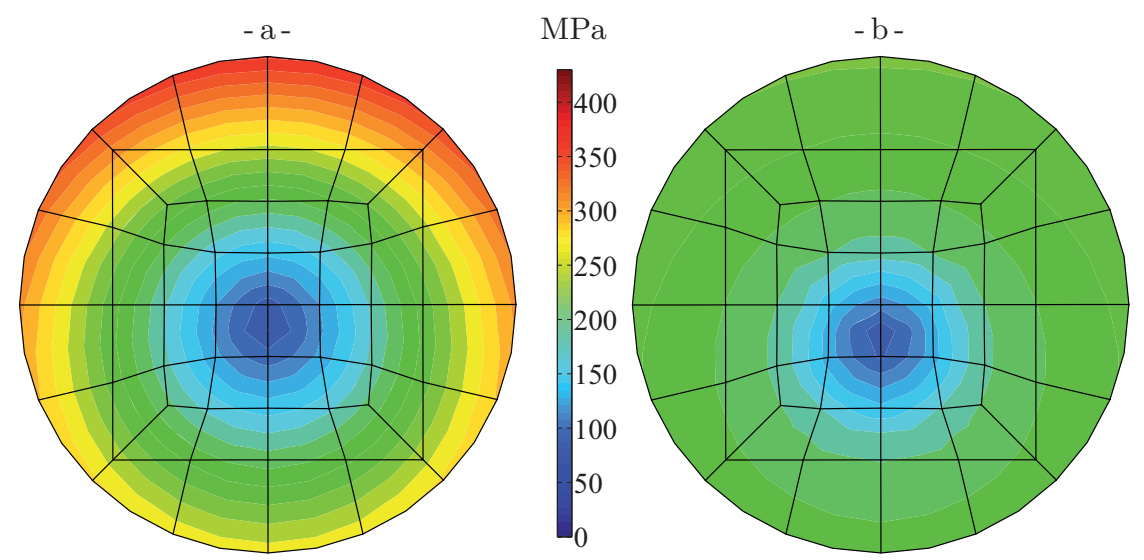

Fig. 10. Distribution of the equivalent stress in a section of the first spring of the SMA/SMA actuator: a) before and b) after cooling. 
The figure 11 shows the distribition of the equivalent stress in a section of the second spring of the SMA/SMA actuator before and after the first cooling. Due to the elongation of the first spring with the appearance of a transformation strain, the equivalent stress decreases in the second one.
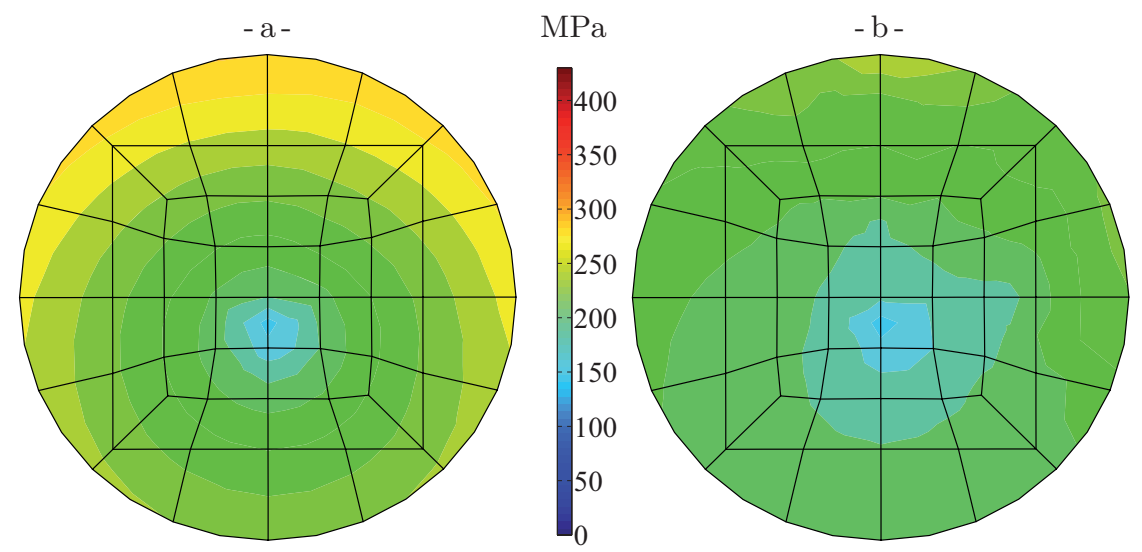

Fig. 11. Distribution of the equivalent stress in a section of the second spring of the SMA/SMA actuator: a) before and b) after cooling.

\section{Conclusion}

In this paper we present a phenomenological model for the thermomechanical behavior of shape memory alloys under nonproportionnal cyclic loadings. It takes into account the specificities of shape memory alloys behavior as the tensile-compresion asymmetry, the return-point memory effect, the reorientation of martensite and the appearance of jammed martensite.

The numerical integration has also been developed. It uses the return-mapping method for forward and reverse transformation. The reorientation is described by alternating forward and reverse transformation during a step.

Simulations have been realised on the structure of an antagonistic springs actuator. This permits to show the performance of the model in a finite element code and to compare the behavior of two different antagonistic springs actuators. The residual strain due to jammed martensite is taken into account but can be quite low on some structures.

This results can be used as a starting point to the study of the fatigue of SMA structures.

\section{References}

1. H. Tobushi, P.H. Lin, T. Hattori, and M. Mikata, JSME International Journal 38(1), 1995 59-67

2. K. Gall and H.J. Maier, Acta Materialia 50, 2002 4643-4657

3. N. Ohno, Materials Science Research International 3(1), 1997 1-9

4. L. Vincent, S. Calloch, and D. Marquis, International Journal of Plasticity 20, 2004 1817-1850

5. F. Auricchio, A. Reali, and U. Stefanelli, International Journal of Plasticity 23, 2007 207-226

6. C. Bouvet, S. Calloch, and C. Lexcellent, European Journal of Mechanics A/Solids 23, 2004 37-61

7. L. Saint-Sulpice, S. Arbab Chirani, S. Calloch, Materials Science \& Engineering A 481-482, 2008 174-177

8. L. Saint-Sulpice, S. Arbab Chirani, S. Calloch, Mechanics of Materials 41, 2009 12-26

9. S. Calloch, K. Taillard, S. Arbab Chirani, C. Lexcellent, and E. Patoor, Materials Science \& Engineering A 438-440, 2006 441-444 